\title{
Anti-epileptic effect of ifenprodil on neocortical pyramidal neurons in patients with malformations of cortical development
}

\author{
XIONGFEI WANG ${ }^{1,2}, \mathrm{XUN} \mathrm{HE}^{3}$, TIANFU $\mathrm{LI}^{4}$, YOUSHENG SHU ${ }^{5}$, SONGTAO QI ${ }^{2}$ and GUOMING LUAN ${ }^{1}$ \\ ${ }^{1}$ Beijing Key Laboratory of Epilepsy, Department of Functional Neurosurgery, Sanbo Brain Hospital, \\ Capital Medical University, Beijing 100093; ${ }^{2}$ Department of Neurosurgery, Nanfang Hospital, Southern Medical University, \\ Guangzhou, Guangdong 510515; ${ }^{3}$ Department of Neurology, Zhujiang Hospital of Southern Medical University, \\ Guangzhou, Guangdong 510280; ${ }^{4}$ Beijing Key Laboratory of Epilepsy, Brain Research Institute, Sanbo Brain Hospital, \\ Capital Medical University, Beijing 100093; ${ }^{5}$ State Key Laboratory of Cognitive Neuroscience and Learning, \\ IDG/McGovern Institute for Brain Research, School of Brain and Cognitive Sciences, The Collaborative \\ Innovation Center for Brain Science, Beijing Normal University, Beijing 100875, P.R. China
}

Received October 19, 2016; Accepted June 22, 2017

DOI: 10.3892/etm.2017.5311

\begin{abstract}
Ifenprodil has been demonstrated to reduce spontaneous action potentials observed by local field potential in animal models. To investigate whether ifenprodil exerts an anti-epileptic effect on neuronal levels in humans, whole-cell patch clamp recordings were used to study the electrophysiological membrane properties of neocortical pyramidal neurons in human brain tissues. Electrophysiological membrane properties and spontaneous spikes of neocortical pyramidal neurons were investigated by using whole-cell patch clamp recordings, prior to and following the application of ifenprodil. In the present study, ifenprodil significantly decreased the membrane input resistance $(\mathrm{P}<0.01)$, membrane time constant $(\mathrm{P}<0.01)$, action potential amplitude $(\mathrm{P}<0.01)$, action potential rising rate $(\mathrm{P}<0.05)$ and falling rate $(\mathrm{P}<0.05)$ on neocortical pyramidal neurons in patients with epilepsy caused by malformations of cortical development (MCD). These results suggested that ifenprodil decreased neuronal excitability of neocortical pyramidal neurons in patients with epilepsy and MCD and demonstrated that ifenprodil may be a potentially specific treatment for refractory epilepsy caused by MCD.
\end{abstract}

\section{Introduction}

Epilepsy is a neurological disorder characterized by the chronic tendency of recurring, unprovoked seizures and $30 \%$ of

Correspondence to: Dr Songtao Qi, Department of Neurosurgery, Nanfang Hospital, Southern Medical University, 1,838 North Guangzhou Avenue, Guangzhou, Guangdong 510515, P.R. China E-mail: qstnfyy@163.com

Key words: ifenprodil, anti-epileptic effect, pyramidal neuron, patients, malformations of cortical development patients have refractory epilepsy (1). For the majority of patients, refractory epilepsy is caused by malformations of cortical development (MCD), which is a heterogenous group of disorders that involves focal cortical dysplasia, tuberous sclerosis and heteropia (2). Furthermore, epileptogenetic focus resection is not suitable for all patients, and previous studies have demonstrated that inflammatory pathways may be targeted as an effective therapeutic strategy in refractory epilepsy $(3,4)$.

Previous studies have supported the promotion of inflammatory and immune processes in epileptogenesis (5), and the majority of them have been described in patients with MCD (6,7). Previous evidence has indicated that the high mobility group box 1 (HMGB1)-Toll-like receptor 4 (TLR4) axis was a proconvulsant pathway in animal models of temporal lobe epilepsy (TLE) (8). Another study showed that, in epileptic human tissues, TLR4 was expressed in astrocytes and dysplastic neurons and HMGB1 was expressed in the glial cytoplasm, which suggested that they may have an indispensable role in human epileptogenesis associated with MCD (6). In addition, HMGB1 has been shown to generate focal seizures by increasing the mean frequency of spontaneous discharges and lowering the ictal event threshold in animal models of TLE (9).

Ifenprodil, which is a selective antagonist of $N$-methyl D-aspartate receptor subtype 2B (NR2B), which contains $\mathrm{N}$-methyl-D-aspartate (NMDA) receptors (10), is able to abrogate the epileptogenetic effect of HMGB1 (8). Despite these findings, the exact effect of ifenprodil on neuronal levels and epileptic network activity remains to be fully elucidated, particularly regarding human neocortical tissues. In the present study, it was investigated whether ifenprodil affected electrophysiological properties and spontaneous spikes in neocortical pyramidal cells (PCs), in addition to the epileptic network activity.

\section{Materials and methods}

Patients. Informed written consent for surgical resection of human tissues for research was obtained from patients or their 
relatives prior to surgery. The handling and use of human brain tissue was approved by the Ethics Committee of Sanbo Brain Hospital, Capital Medical University (Beijing, China). The study included a total of 6 patients enrolled between October 2014 and December 2015 (males, $n=5$; females, $n=1$; age,

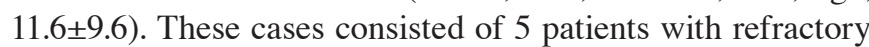
epilepsy with the following inclusion criteria: i) Confirmed diagnosis of refractory epilepsy; and ii) pathological diagnosis of malformations of cortical development. Patients who had undergone radiotherapy or chemotherapy were excluded from the study. There was 1 case of glioma without epilepsy also included. Each patient received cortical focal resection. For each case, at least 2 PCs were recorded. The diagnosis of each patient was made by a multidisciplinary team according to the International League Against Epilepsy classification system (11).

Clinical information. The standardized information obtained from patients included clinical history evaluation, routine neurological examinations, such as motor, sensor, vision, language and auditory sense examinations and scalp electroencephalogram (EEG) video-recordings, magnetic resonance imaging (MRI) and postoperative computed tomography. Clinical history was obtained from medical records and included the following: Age at seizure onset, age at surgery, clinical manifestation, type of surgery (multi-lobar or lobar/focal), side of operation, sex, seizure frequency, history of antiepileptic drugs (AEDs) and pathology examination described previously (12), as indicated in Table I. In particular, the region of brains sampled for in vitro electrophysiological studies were recorded.

Electrophysiological methods. Human brain tissues, chosen as epileptic onset zone according to preoperative evaluation (2/6 with high frequency oscillations in stereotactic electroencephalogram [SEEG]), were excised to investigate the electrophysiological character of cortical neurons.

Tissues were removed microsurgically and directly placed in ice-cold slicing solution containing $26 \mathrm{mM} \mathrm{NaHCO}$, $2.5 \mathrm{mM} \mathrm{KCl}, 5 \mathrm{mM} \mathrm{MgCl}{ }_{2}, 1.25 \mathrm{mM} \mathrm{NaH}_{2} \mathrm{PO}_{4}, 10 \mathrm{mM}$ dextrose, $213 \mathrm{mM}$ sucrose, $1.0 \mathrm{mM} \mathrm{CaCl}_{2}$ and $1.0 \mathrm{mM} \mathrm{MgSO}_{4}$ ( $\mathrm{pH}$ 7.2-7.4). Within 1-2 h, slices $350 \mu \mathrm{m}$ thick were cut using a Leica slicer and incubated in regular artificial cerebrospinal fluid (ACSF) containing $126 \mathrm{mM} \mathrm{NaCl}, 2.5 \mathrm{mM} \mathrm{KCl}, 1.25 \mathrm{mM}$ $\mathrm{NaH}_{2} \mathrm{PO}_{4}, 26 \mathrm{mM} \mathrm{NaHCO}, 25 \mathrm{mM}$ Dextrose, $2 \mathrm{mM} \mathrm{MgSO}$

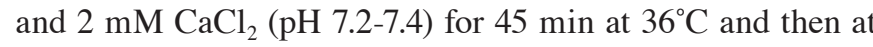
room temperature until use.

During recordings, slices, without the cover slip, from the original and ifenprodil groups were perfused at $35-36^{\circ} \mathrm{C}$ at $3 \mathrm{ml} / \mathrm{min}$ for $2 \mathrm{~h}$ in $\mathrm{Mg}^{2+}$-free modified ACSF composed of $126 \mathrm{mM} \mathrm{NaCl}, 3.5 \mathrm{mM} \mathrm{KCl}, 1.25 \mathrm{mM} \mathrm{NaH}{ }_{2} \mathrm{PO}_{4}, 26 \mathrm{mM}$ $\mathrm{NaHCO}_{3}, 25 \mathrm{mM}$ dextrose and $2 \mathrm{mM} \mathrm{CaCl}_{2}(\mathrm{pH}$ 7.2-7.4) to mimic epileptic activity $(13,14)$. Whole-cell recordings were achieved using glass pipettes (3-5 M 2 ) filled with $140 \mathrm{mM}$ K-Gluconate, $3 \mathrm{mM} \mathrm{KCl}, 2 \mathrm{mM} \mathrm{MgCl}_{2}, 10 \mathrm{mM}$ HEPES, 0.2 mM EGTA, $2 \mathrm{mM} \mathrm{Na}{ }_{2}$ ATP and $0.2 \%$ biocytin ( $\mathrm{pH}$ 7.25-7.30; osmolality, 280-290 mOsm) for voltage and current clamp recordings. Spontaneous spikes lasting for at least $2 \mathrm{~min}$, action potential induced by depolarizing currents (40 pA current increment; $500 \mathrm{msec}$ ) and membrane potential
(Vm) responses to hyperpolarized current (-100 pA; $500 \mathrm{msec})$ or a series of current steps (500 msec in duration) were recorded, respectively, to compare differences of intrinsic electrophysiological properties prior to and after the application of ifenprodil (5 $\mu \mathrm{M}$; I2892; Sigma-Aldrich; Merk KGaA, Darmstadt, Germany).

The five patients with epilepsy underwent epileptogenic zone resections and the patient without epilepsy underwent tumor resection. Subsequently, patients with epilepsy were split into the following groups: i) According to video EEG recordings, patients were divided into a high frequency oscillations (HFOs) group ( $\mathrm{n}=3$ patients) and a non-HFOs ( $\mathrm{n}=2$ patients) group; and according to pathological results, ii) patients with focal cortical dysplasia type II and tuberous sclerosis (TS) were classed as the TS complex (TSC)-related group ( $n=3$ patients) and iii) patients with focal cortical dysplasia type I and type IIId were classed as the idiopathic group ( $n=2$ patients). The patient without epilepsy was classed as the non-epilepsy group.

For all brain tissue obtained from the 6 patients enrolled in the current study, whole-cell recordings on pyramidal-shaped neurons were performed and interneuron-shaped neurons under the DIC (differential interference contrast) microscope (x400) were avoided due the focus of the study being on excitatory modulation. According to our previous study, PCs and inhibitory interneurons were identified by their firing patterns (15). Depolarizing currents were applied (40 pA current increment; $500 \mathrm{msec}$ ) to induce action potentials, and all cells were recorded at resting membrane potential. Once the recordings were established, the type of neuron was re-confirmed using staining techniques, after having identified them by firing patterns.

Hemtoxylin and eosin staining. Tissue samples from all 6 patients were fixed in $10 \%$ formalin at room temperature for $24 \mathrm{~h}$ and embedded in paraffin prior to being cut into sections $4 \mu \mathrm{m}$ thick. Sections were then mounted on pre-coated glass slides (Star Frost, Waldemar Knittel GmbH, Braunschweig, Germany). Sections of all specimens were stained with hematoxylin and eosin at room temperature for $4 \mathrm{~h}$, and images were visualized using a Leica microscope, captured using an Optronics DEI-750 three-chip camera equipped with a BQ 8000 sVGA frame grabber (x400; Optronics, Goleta, CA, USA) and analyzed using Bioquant software 2011 (Bioquant Image Analysis Corporation, Nashville, TN, USA).

Drug treatment. For all slices obtained from patients enrolled in the current study, including the epilepsy and non-epilepsy groups, Vm responses to the hyperpolarized current, spontaneous spiking and $\mathrm{Vm}$ responses to serials of hyperpolarized current steps were recorded. Following original recordings for $20 \mathrm{~min}$, brain tissue sections from all 6 patients were immersed in $5 \mu \mathrm{M}$ ifenprodil for $5 \mathrm{~min}$ in $36^{\circ} \mathrm{C}$ and the above-mentioned parameters were subsequently examined again. Slices were then fixed in $4 \%$ paraformaldehyde for $12 \mathrm{~h}$ at room temperature for subsequent avidin staining.

Histological procedures. Alexa Fluor $488(200 \mu \mathrm{M})$ and $0.2 \%$ biocytin were added to internal solution (K-Gluconate $140 \mathrm{mM}$, KCl $3 \mathrm{mM}, \mathrm{MgCl} 2 \cdot 6 \mathrm{H} 2 \mathrm{O} 2 \mathrm{mM}$, HEPES $10 \mathrm{mM}$, EGTA $10 \mathrm{mM}$, Na2ATP $2 \mathrm{mM}, 285 \mathrm{mOsm}, \mathrm{pH}$ 7.3) as 


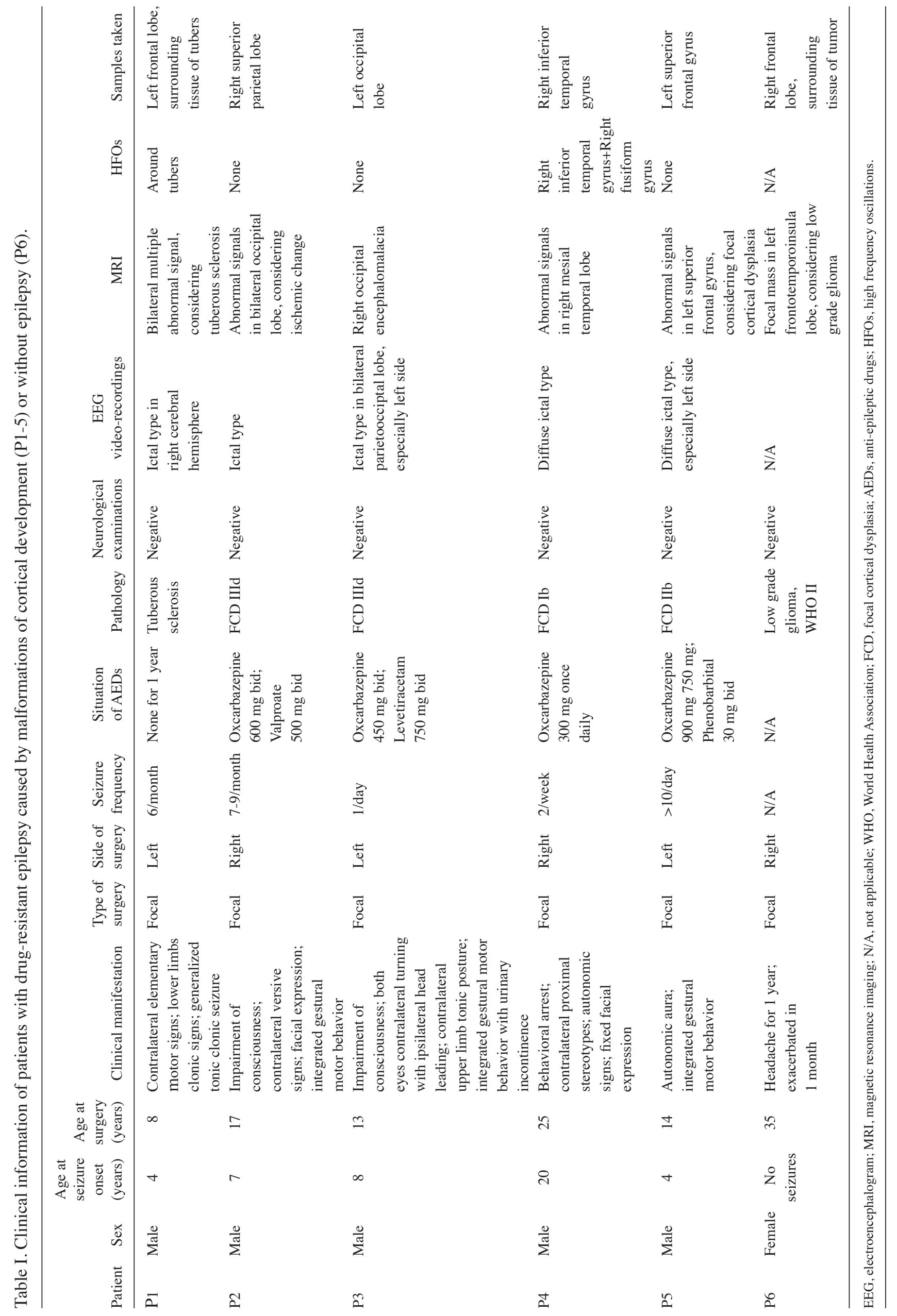


described previously (15) in order to study and record labeled cells. Following the completion of recordings by patch clamp, 3 human brain slices from the TSC, idiopathic and non-epilepsy groups were fixed in $8 \%$ PFA at room temperature and $8 \%$ sucrose (w/v) in $0.1 \mathrm{Mmol}$ PBS (pH 7.4) for $1 \mathrm{~h}$. Slices were rinsed in $0.01 \mathrm{Mmol}$ PBS 3 times, and subsequently incubated in $0.5 \%$ Triton $\mathrm{X}-100$ for $0.5 \mathrm{~h}$ at room temperature and $10 \%$ bovine serum albumin (Sigma-Aldrich; Merck KGaA, Darmstadt, Germany) was used as a blocking solution at room temperature for $1 \mathrm{~h}$. Once slices were washed in PBS 3 times for $10 \mathrm{~min}$, they were incubated in strept-avidin (1:1,000; cat no. SA-5000, Vector Laboratories, Inc., Burlingame, CA, USA) for $2 \mathrm{~h}$ at room temperature. Confocal images were taken with a laser scanning confocal microscope (Nikon A1; Nikon Corporation; Tokyo, Japan) with a x40 objective. $\mathrm{Z}$-stack images of labeled cells were attained with an interval of $0.75 \mu \mathrm{m}$.

Statistical analysis. All of the electrophysiological values were presented as mean \pm standard error and all experiments were repeated in triplicate. For two independent observations, a two-sample Student's t-test was used for normally-distributed data, whereas non-normal data were compared using Wilcoxon rank-sum test. For multiple observations a one-way ANOVA followed by a Student-Newman-Keuls test was used to examine the significance between groups with a normal distribution, whereas when data did not have a normal distribution a Kruskal-Wallis $\mathrm{H}$ test was used. $\mathrm{P}<0.05$ was considered to indicate a statistically significant difference. Passive parameters were derived from $\mathrm{Vm}$ responses to current steps.

Input resistance was calculated using the following formula: Steady state Vm changes/current amplitudes. Action potential (AP) amplitude was considered the voltage value between the threshold and peak. AP threshold was the voltage value at the time when the AP rising slope reached $20 \mathrm{~V} / \mathrm{sec}$. AP half-width was determined as the AP duration at half amplitude. AP rising rate was the highest value of voltage-time slope in the AP rising phase. To detect the AP falling rate, the lowest value of the voltage-time slope in the AP falling phase was used. Frequency-current intensity curve (F-I curve) indicated the association between serials of hyperpolarized currents and the number of spikes they evoked.

Every random spontaneous excitatory input was identified, including subthreshold depolarizations and spontaneous spikes, and then considered all-or-none random spontaneous bursts into an event. In the statistical analysis, excitatory inputs with an amplitude $>15 \mathrm{mV}$ were included. For each cell, the induced spontaneous spikes and events within the selected $120 \mathrm{sec}$ were measured.

All analysis was performed using Matlab (R2014a, The Math Works; Natick, MA, USA), SPSS 21 for Mac (IBM Corp., Armonk, NY, USA) and Spike2 (7.11a; Cambridge electronic design; Cambridge, UK).

\section{Results}

Patient clinical information. Clinical information is presented in Table I. Focal lesions were localized by clinical manifestation, preoperative MRI (Fig. 1A) and SEEG (data not shown). Following the combination of postoperative CT and
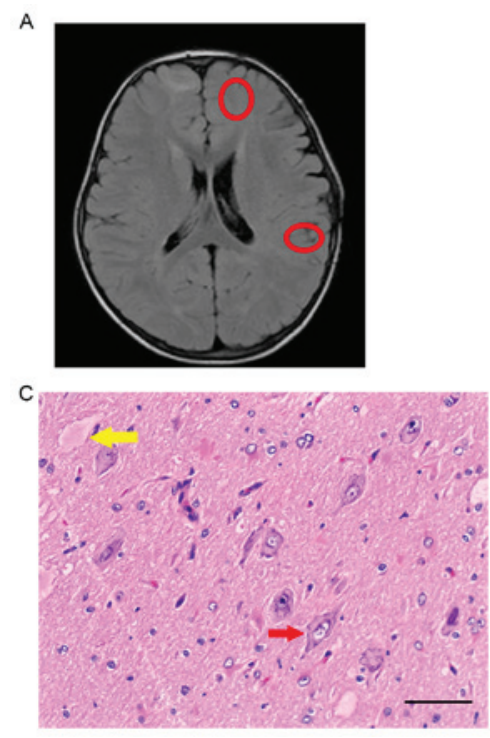

Figure 1. Example of human brain tissue. (A) P1 was an 8 year old male patient diagnosed with refractory epilepsy caused by tuberous sclerosis 4 years prior to the start of the current study. Fluid-attenuated inversion recovery weighted magnetic resonance imaging revealed tubers in left frontal lobe (red circles). (B) Postoperative computed tomography revealed the exact location of the human tissue for study (red circles). (C) Pathological examination of HE staining showed dysmorphic neuron (red arrow) and balloon cells (yellow arrow). Scale bar, $50 \mu \mathrm{m}$. (D) Example of recording pyramidal neuron using a differential interference contrast microscope. Magnification, x400.

pathological results, the exact locations of samples were studied and dysmorphic neurons and balloon cells were noted in Fig. 1B and C). The morphology of a pyramidal neuron is presented in Fig. 1D.

Investigation of electrophysiological properties of neocortical $P C$ s in patients with MCD. To identify the correlation between clinical manifestations and PC electrophysiological properties, patients with focal cortical dysplasia type II and tuberous sclerosis (TS) were categorized as the TS complex (TSC)-related group, and patients with focal cortical dysplasia type I and type IIId were classed as the idiopathic group. According to video EEG recordings, patients were divided into the HFOs group $(n=3)$ and non-HFOs $(n=2)$ group. In addition, one patient without epilepsy was selected as the non-epilepsy group.

Electrophysiological properties of neocortical PCs in patients with MCD were determined (Fig. 2). The morphology of recorded pyramidal neurons, which were similar to those under physiological condition, were indicated in Fig. 2A. The rising and falling rate of AP in the TSC-related group was significantly faster than those in the idiopathic and non-epilepsy groups (Fig. 2C and D, respectively; $\mathrm{P}<0.01$ ). The half-width of APs in the TSC-related group was significantly decreased compared with those in idiopathic and non-epilepsy groups (Fig. 2B; $\mathrm{P}<0.01$ ), whereas there was no significant difference in the membrane input resistance, membrane capacitance, time constant, amplitude of AP, and threshold potential among the 3 groups (data not shown).

The characteristics determined by EEG indicated there was no significant difference in the membrane input resistance, membrane capacitance, time constant, AP rising rate, threshold potential, amplitude of AP, half-width of AP and AP 

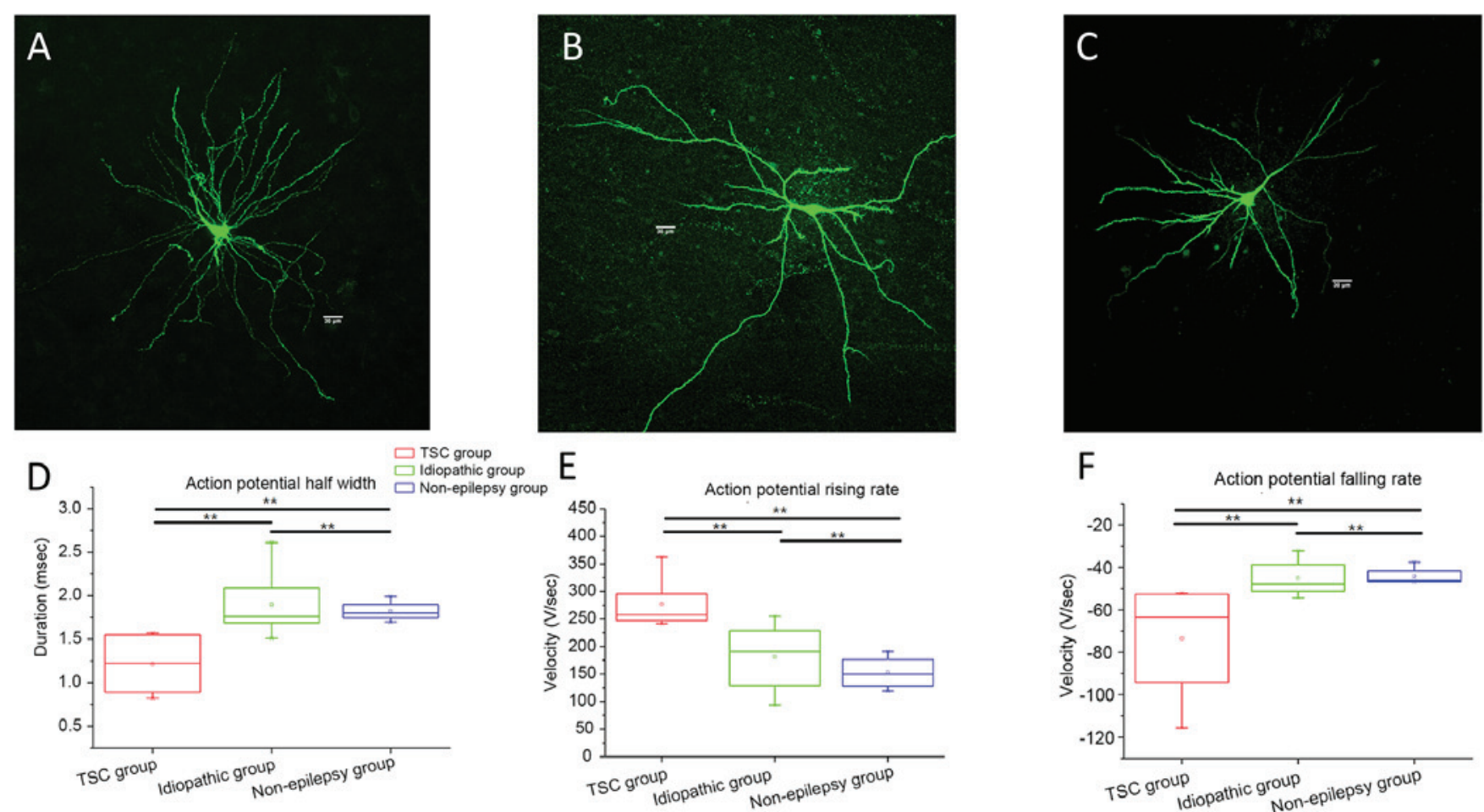

Figure 2. Investigation of electrophysiological properties of neocortical pyramidal cells in patients with malformations of cortical development. (A-C) Neocortical pyramidal cells, from the TSC group (A), idiopathic group (B) and non-epilepsy group (C), were stained with avidin staining. Scale bar, $30 \mu \mathrm{m}$. (D) Action potential half-width in the TSC-related group ( $\mathrm{n}=6$ cells) was decreased compared with that in idiopathic group ( $\mathrm{n}=8$ cells) and non-epilepsy group ( $n=4$ cells) (Student-Newman-Keuls test; $\left.{ }^{* * *} \mathrm{P}<0.01\right)$. (E and F) In aspect of pathology, action potential rising and falling rates in the TSC group ( $=6$ cells) were significantly faster compared with those in the idiopathic $\left(\mathrm{n}=8\right.$ cells) and non-epilepsy groups ( $\mathrm{n}=4$ cells) (Student-Newman-Keuls test, $\left.{ }^{* *} \mathrm{P}<0.01\right)$. Box-and-whisker plots present the range, interquartile range and median; 'o' indicates the mean value. TSC, tuberous sclerosis complex.

falling rate between the HFOs group and non-HFOs group (data not shown).

Modulation of electrophysiological properties in PCs by ifenprodil. As HMGB1 modulates the excitability of PCs by activating NR2B-containing NMDA receptors (16), the present study examined the exact electrophysiological changes in PCs. Changes in whole-cell membrane electrophysiological properties were obtained by injecting hyperpolarizing and depolarizing currents steps, respectively. Hyperpolarizing currents $(-100 \mathrm{pA} ; 500 \mathrm{msec})$ were applied to the recording cell to examine passive membrane properties. Subsequently, depolarizing currents (40 pA current increment; $500 \mathrm{msec}$ ) were applied to induce action potentials. Cells were recorded at resting membrane potential.

Electrophysiological membrane properties for each cell were compared, including input resistance, time constant, capacitance, AP amplitude, AP half-width, AP threshold, AP rising rate and falling rate (Table II). Among patients with epilepsy, ifenprodil significantly decreased the input resistance and time constant compared with the original group (Fig. 3A; $\mathrm{P}<0.01$ ), whereas no significant difference was indicated in capacitance (Table II; $\mathrm{P}=0.94$ ). Furthermore, ifenprodil treatment significantly decreased AP amplitude compared with the original group ( $\mathrm{P}<0.01$; Table II; Fig. 3B), but no significant alteration in AP half-width was observed (Table II; Fig. 3B), which likely indicated decreased $\mathrm{Ca}^{2+}$ entry following application of ifenprodil $(17,18)$. Additionally, AP rising and falling rates were significantly decreased following ifenprodil treatment compared with the original group, respectively (Table II; Fig. 3B; P<0.05). However, the AP threshold showed no significant difference prior to and after application of ifenprodil. In patients without epilepsy, no significant differences were noted in the membrane properties prior to and after application of ifenprodil (Table II). In addition, no significant difference in the membrane properties between patients with and without epilepsy were exhibited, with one exception; significantly increased membrane capacitance was detected in epilepsy patients (Table II; $\mathrm{P}<0.05$ ).

To investigate the modulation of neuronal excitability of PCs by ifenprodil, a series of depolarizing current steps were applied to generate an F-I curve. Fig. 4A indicates an example of the voltage response of recording PCs under the current stimulus with the same intensity prior to and after application of ifenprodil. Results suggested that in the epilepsy group, ifenprodil decreased the mean number of spikes compared with the original group; however, this was not a statistically significant difference (Fig. 4B). Conversely, the mean number of spikes was increased following the application of ifenprodil in non-epilepsy patients, although this was not a statistically significant difference (Fig. 4B). These results indicate that ifenprodil may decrease neuronal excitability of PCs in patients with epilepsy.

Modulation of epileptic network activity by ifenprodil. To mimic epileptic activity, the brain tissues were perfused in $\mathrm{Mg}^{2+}$-free ACSF instead of normal ACSF. A total of $85.7 \%$ of slices (12/14) generated epileptiform activity in cortical tissue 

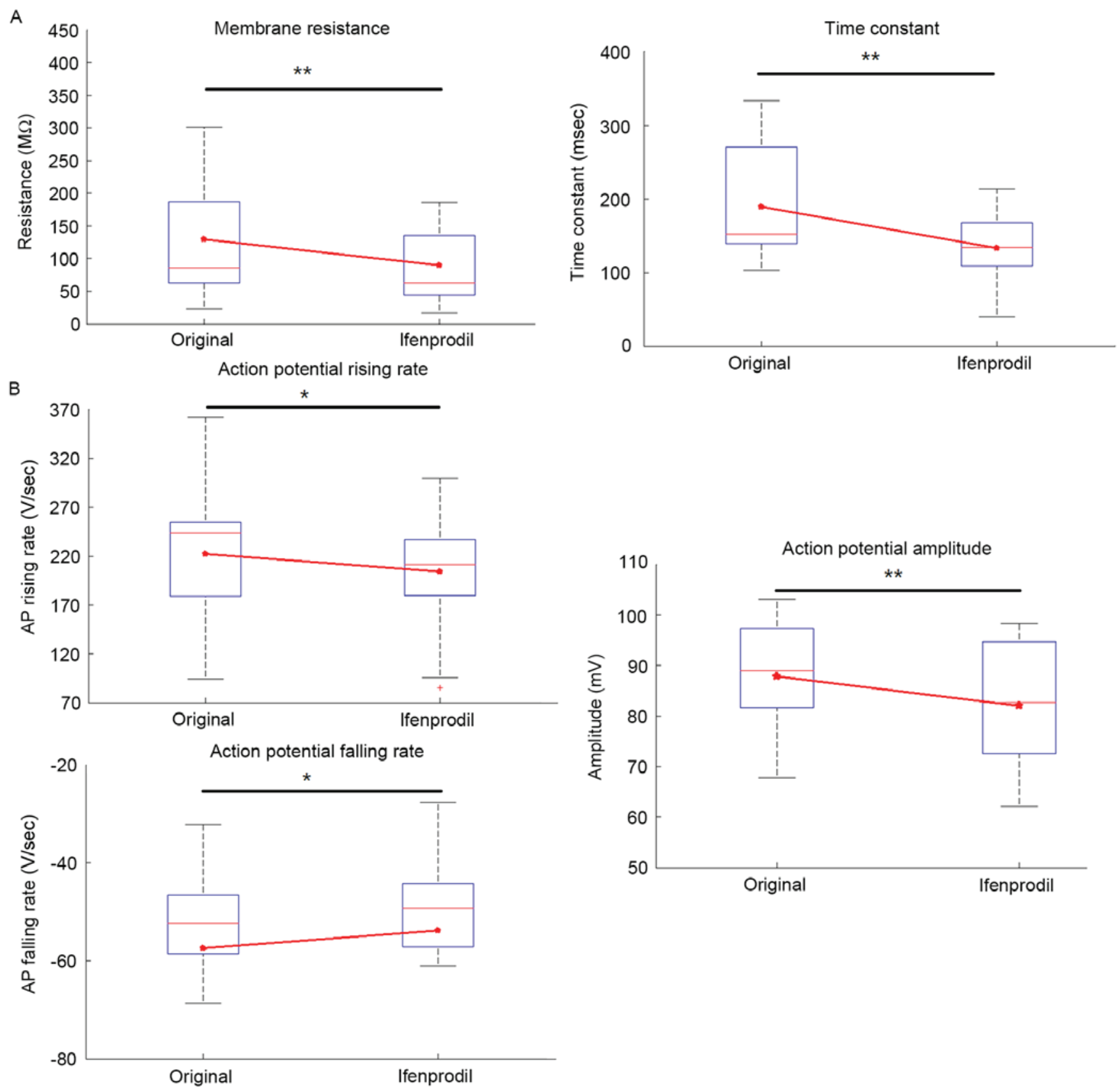

Figure 3. Modulation of electrophysiological membrane properties in neocortical pyramidal cells. (A) Membrane resistance and time constant were significantly decreased compared with the original group following the application of $5 \mu \mathrm{M}$ ifenprodil. (Wilcoxon matched-pairs signed-rank test; ${ }^{* *} \mathrm{P}<0.01 ; \mathrm{n}=14$ cells) (B) Ifenprodil significantly decreased AP amplitude (Wilcoxon matched-pairs signed-rank test; * $\mathrm{P}<0.01 ; \mathrm{n}=14$ cells), AP rising rate (two-tailed paired $\mathrm{t}$-test; ${ }^{\mathrm{P}} \mathrm{P}<0.05 ; \mathrm{n}=14$ cells) and AP falling rate (Wilcoxon matched-pairs signed-rank test; $\mathrm{P}<0.05 ; \mathrm{n}=14$ cells) compared with the original group, respectively. Box-and-whisker plots present the range, interquartile range and median; red points indicate the mean value. AP, action potential.

obtained from epilepsy patients. Once a stable epileptic network activity was achieved in 5 slices for $\geq 25$ min, ifenprodil was applied and the generation of epileptiform activity was decreased after applying ifenprodil (Fig. 5). The number of spikes for each depolarized event was decreased in the Ifenprodil group compared with the original group (Fig. 5A). As defined above, depolarizing potentials with amplitude $>15 \mathrm{mV}$ and duration $>300 \mathrm{msec}$ were considered as an event. Bath application of $5 \mu \mathrm{M}$ ifenprodil significantly decreased the number of depolarizing events compared with the original group (Fig. 5D; $\mathrm{P}<0.05$ ) and significantly increased the inter-burst-interval (Fig. 5C; $\mathrm{P}<0.05)$. Furthermore, the frequency distribution plot indicated that ifenprodil significantly reduced the quantity of depolarizing events in every $10-\mathrm{Hz}$ step within the selected $120 \mathrm{sec}$ by (Fig. 5B; $\mathrm{P}<0.05$ ).

\section{Discussion}

Immune mediators are functional in the immune response to infection; however, recent studies have demonstrated that they also have alternative roles $(19,20)$. Increasing experimental evidence has demonstrated that immune mediators are associated with neuromodulation (21). In the last two decades, findings have suggested that immune mediators, including 
Table II. Modulation of electrophysiological properties of pyramidal neurons.

\begin{tabular}{|c|c|c|c|c|c|c|c|c|c|c|}
\hline \multirow[b]{3}{*}{ Property } & \multicolumn{5}{|c|}{ Epilepsy } & \multicolumn{5}{|c|}{ Non-epilepsy } \\
\hline & \multicolumn{2}{|l|}{ Original } & \multicolumn{2}{|l|}{ Ifenprodil } & \multirow[b]{2}{*}{ P-value } & \multicolumn{2}{|l|}{ Original } & \multicolumn{2}{|l|}{ Ifenprodil } & \multirow[b]{2}{*}{ P-value } \\
\hline & Mean \pm SE & $\mathrm{n}$ & Mean \pm SE & $\mathrm{n}$ & & Mean \pm SE & $\mathrm{n}$ & Mean \pm SE & $\mathrm{n}$ & \\
\hline \multicolumn{11}{|l|}{$\begin{array}{l}\text { Passive membrane } \\
\text { property }\end{array}$} \\
\hline Resistance $(\mathrm{M} \Omega)$ & $140.665 \pm 29.768$ & 14 & $102.683 \pm 21.365$ & 14 & $<0.01$ & $191.297 \pm 22.947$ & 4 & $218.875 \pm 15.168$ & 4 & 0.144 \\
\hline Tau (msec) & $18.923 \pm 2.091$ & 14 & $13.295 \pm 1.208$ & 14 & $<0.01$ & $19.553 \pm$ SE 3.943 & 4 & $21.752 \pm 3.236$ & 4 & 0.465 \\
\hline Capacitance (pf) & $189.267 \pm 36.891$ & 14 & $188.007 \pm 31.819$ & 14 & 0.778 & $101.774 \pm 12.895$ & 4 & $98.112 \pm 10.180$ & 4 & 0.715 \\
\hline AP amplitude (mV) & $87.999 \pm 3.332$ & 14 & $83.043 \pm 3.777$ & 14 & $<0.01$ & $78.699 \pm 3.881$ & 4 & $76.352 \pm 4.354$ & 4 & 0.715 \\
\hline AP half width (msec) & $1.605 \pm 0.134$ & 14 & $1.615 \pm 0.152$ & 14 & 0.221 & $1.8223 \pm 0.0600$ & 4 & $1.871 \pm 0.124$ & 4 & 0.715 \\
\hline \multicolumn{11}{|l|}{$\begin{array}{l}\text { Active membrane } \\
\text { property }\end{array}$} \\
\hline AP threshold (mV) & $-43.549 \pm 3.100$ & 14 & $-44.327 \pm 2.594$ & 14 & 0.778 & $-36.885 \pm 1.1450$ & 4 & $-36.030 \pm 2.829$ & 4 & 0.465 \\
\hline AP rising rate $(\mathrm{V} / \mathrm{sec})$ & $222.037 \pm 19.260$ & 14 & $203.949 \pm 16.438$ & 14 & $<0.05$ & $152.413 \pm 15.599$ & 4 & $144.990 \pm 13.998$ & 4 & 0.715 \\
\hline AP falling rate $(\mathrm{V} / \mathrm{sec})$ & $-57.454 \pm 6.013$ & 14 & $-53.819 \pm 5.414$ & 14 & $<0.05$ & $-44.219 \pm 2.205$ & 4 & $-43.360 \pm 3.507$ & 4 & 0.715 \\
\hline
\end{tabular}

Statistical significance of differences was evaluated via Wilcoxon test, with the exception of AP rising rate, which was tested using a paired two sample t-test. AP; action potential; SE, standard error.

A

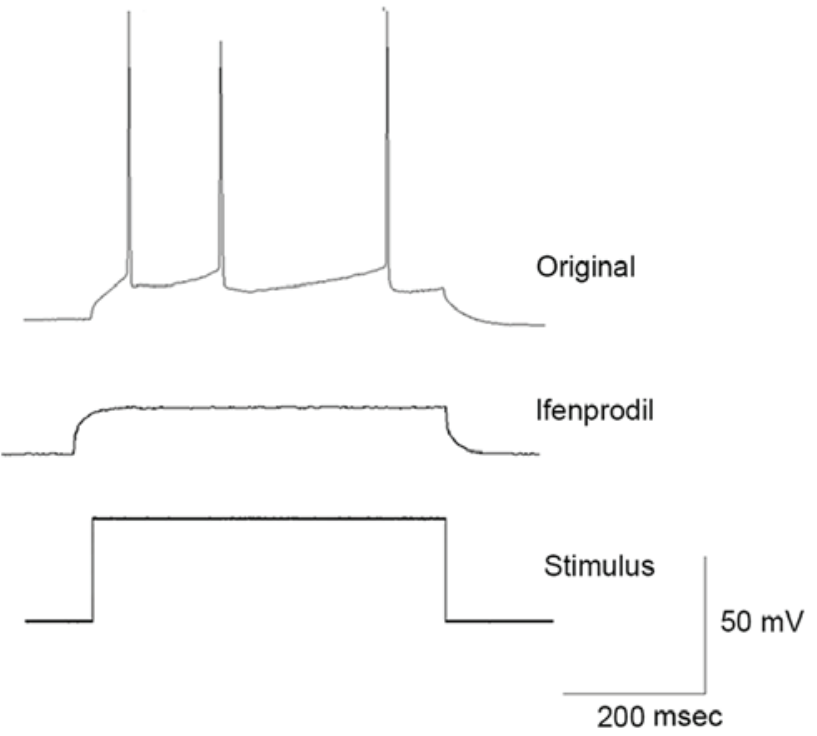

B

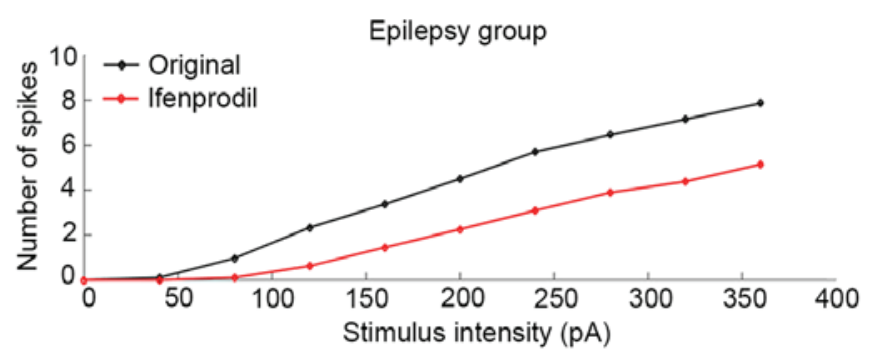

Control group

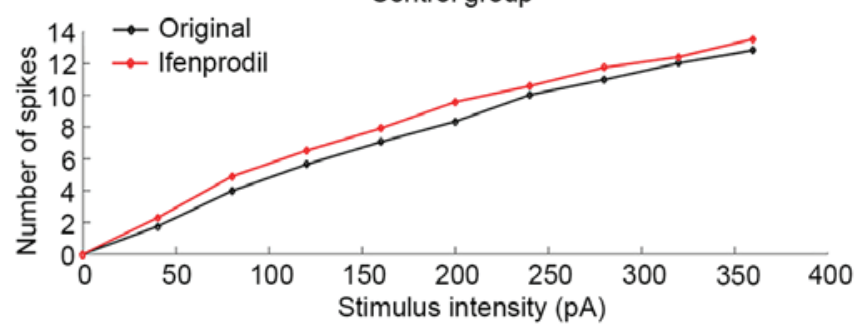

Figure 4. Modulation of neural excitability. (A) Voltage response of a neocortical pyramidal cell recording under current stimulus with the same intensity prior to and after application of $5 \mu \mathrm{M}$ ifenprodil. (B) For patients with epilepsy, the frequency-current intensity curve indicated that ifenprodil reduced the mean number of spikes; however, this was not statistically significant. Conversely, the mean number of spikes was increased following the application of $5 \mu \mathrm{M}$ ifenprodil in patients without epilepsy, although this was not statistically significant. $\mathrm{P}=0.098$ and $\mathrm{P}=0.534$, respectively; $\mathrm{n}=14$ cells.

pathogen associated molecular patterns, danger associated molecular patterns (DAMPs) and extracellular matrix components, modulate voltage-dependent ion channels, receptor-dependent ion channel and synaptic strength (22). HMGB1, which is a type of DAMP, was expressed in the nucleus in both neurons and glial cells under normal physiological conditions, and is produced from cell necrosis or active release following stimulation (23). In an animal model, the HMGB1/TLR4 axis increased $\mathrm{Ca}^{2+}$ influx through phosphorylation of the NMDA-NR2B receptor (16). The present study revealed that AP amplitude in PC was significantly decreased following the application of ifenprodil in the epilepsy group, which indicated a reduction in $\mathrm{Ca}^{2+}$ influx (17). However, $\mathrm{Ca}^{2+}$ channels are categorized in three families and mediate different types of currents (24); therefore, further investigation is required to identify which family was associated with this effect. As $\mathrm{Ca}^{2+}$ influx is necessary to activate the release of HMGB1 (25), it was postulated that ifenprodil likely decreased the release of HMGB1 in PCs. HMGB1 also provokes astrocytes to release immune mediators (26), and application of ifenprodil potentially decreases the release of a variety of immune mediators.

As HMGB1 and NMDA receptors are upregulated in the nidus of the neuron among patients with MCD (27), it was 
A

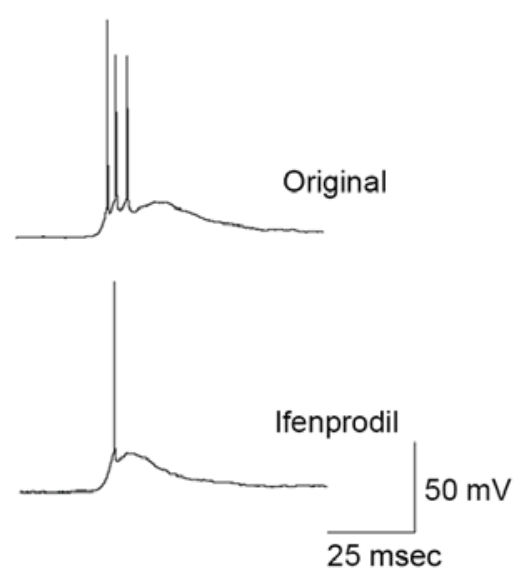

Interval time

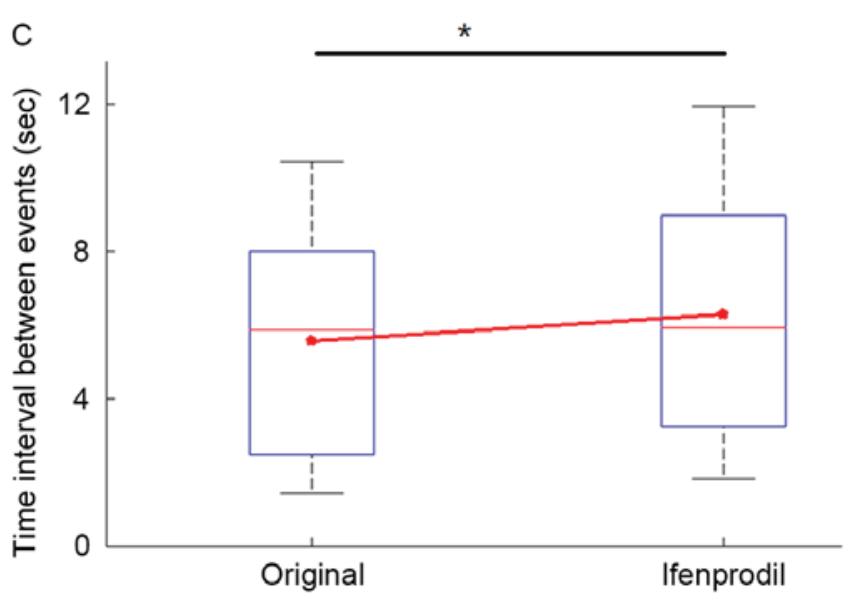

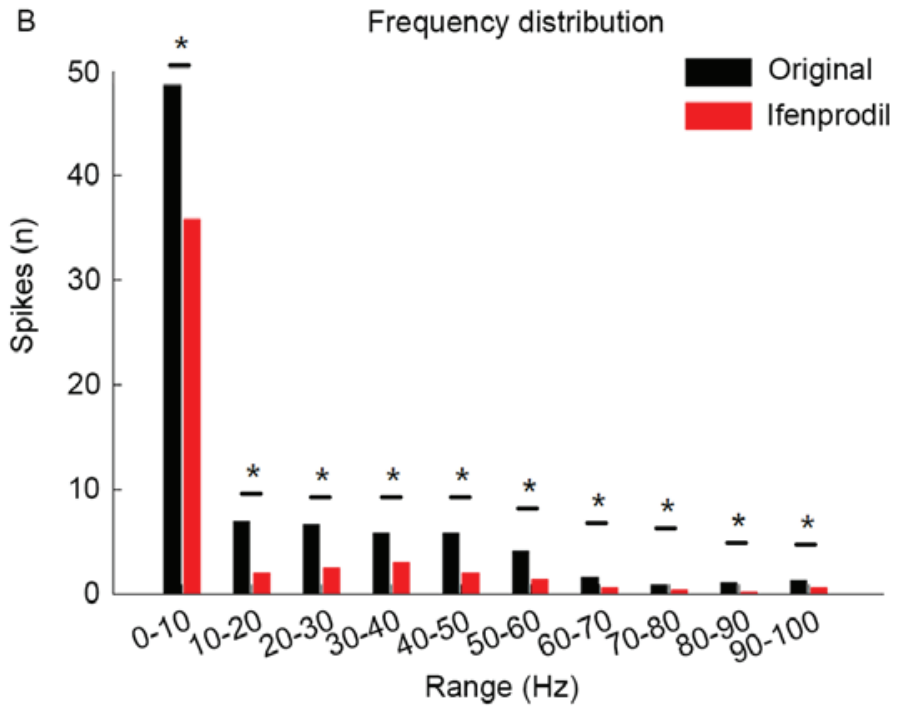

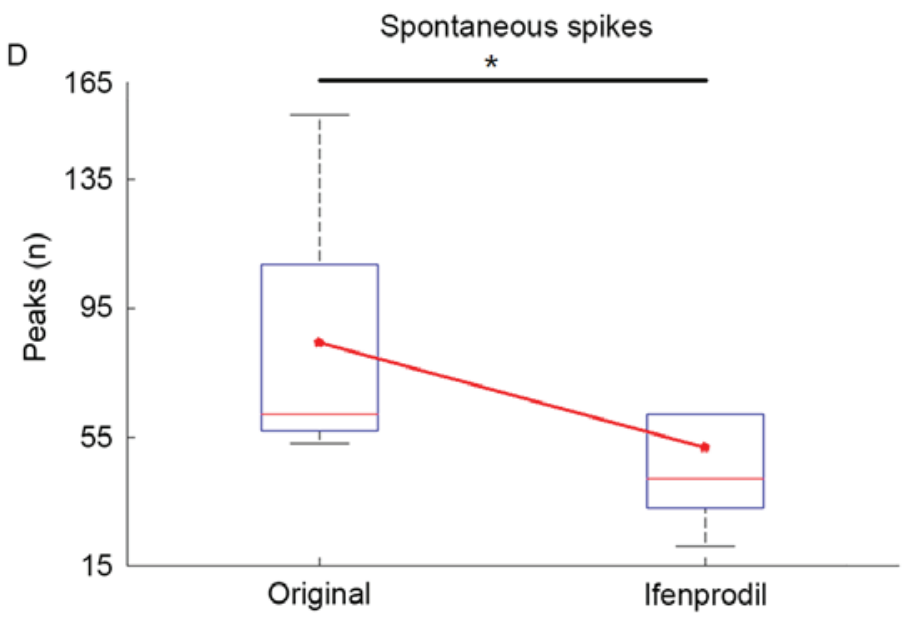

Figure 5. Modulation of spontaneous spikes. (A) Spontaneous spikes of a neocortical pyramidal cell recording prior to and after application of $5 \mu \mathrm{M}$ ifenprodil. (B) Frequency distribution determined the application of ifenprodil reduced the quantity of depolarizing events in every $10-\mathrm{Hz}$ step within the selected 120 sec. ${ }^{*} \mathrm{P}<0.05$. (C and $\left.\mathrm{D}\right)$ Application of $5 \mu \mathrm{M}$ ifenprodil significantly decreased the quantity of depolarizing events (Wilcoxon matched-pairs signed-rank test; ${ }^{*} \mathrm{P}<0.05 ; \mathrm{n}=5$ slices) and significantly the increased inter-burst-interval (two-tailed paired $\mathrm{t}$-test; ${ }^{*} \mathrm{P}<0.05 ; \mathrm{n}=5$ slices) compared with the original group within the selected $120 \mathrm{sec}$.

expected that application ifenprodil would exhibit pathological-specific modulation of neuronal excitability in PCs. In the present study, the application of ifenprodil reduced neuronal excitability of PCs in patients with epilepsy, but marginally enhanced PC neuronal excitability in patients without epilepsy. Coincidentally, application of ifenprodil significantly decreased membrane input resistance in patients with epilepsy. Previous studies showed that NR2B was predominantly expressed in the extrasynaptic region (28) and extrasynaptic neurotransmitter receptor activation appeared to modulated membrane potential (29). These findings suggest that NR2B blockage by application of ifenprodil may hyperpolarize membrane potential, which results in an increase in membrane conductance by HCN channel activation (30).

Neuronal excitability was determined by AP characteristics and spiking pattern, thereby neuronal excitability should be described by both AP shape and F-I curve alteration. The present results indicated that application of ifenprodil altered the AP characteristics in the epilepsy group by decreasing either $\mathrm{AP}$ amplitude, AP rising rate or AP falling rate. These findings suggested that the application of ifenprodil likely decreased $\mathrm{Ca}^{2+}$ influx during the generation of APs, and decreased the conductance of $\mathrm{Ca}^{2+}$-dependent $\mathrm{K}^{+}$channel in AP falling phase (31). Using calcium imaging in an animal model, a previous study demonstrated that HMGB1 increased spontaneous ictal-like discharges, which were blocked by tetrodotoxin, which is a voltage-gated sodium channel blocker (9). The present study showed that application of ifenprodil decreased the total number of spontaneous spikes and increased inter-burst-interval time among PCs in patients with epilepsy.

With the exception of HMGB1, experimental evidence has revealed interactions between interleukin-1 $\beta$ and NR2B subunits in hippocampal neurons (32). The interaction between HMGB1 and IL1 $\beta$ also exhibited amplification of the inflammatory response in osteoarthritis (33). We subsequently speculated that the application of ifenprodil likely had a potent anti-epileptic effect via the direct blockage of HMGB1/TLR4 axis (8).

In clinical practice, antiepileptic agents have non-specific effects on preventing epileptic activity by decreasing 
excitation or increasing the inhibition in every neuron within the cortex $(34,35)$. In patients with epilepsy and MCD, the expression of HMGB1 was upregulated in dysmorphic neurons and translocated from the nucleus to the cytoplasm in glial cells in a previous study (27). No similar findings in patients without epilepsy were identified in the present study. In addition, animal experiments have revealed that the activation of HMGB1/TLR4 axis also has long term effects on epileptogenesis by reducing the epileptogenetic threshold (36).

Ifenprodil has been proven to be safe for humans in the treatment of ischemic brain injury $(37,38)$ as a non-competitive and highly selective antagonist of NMDA receptors containing the NR2B subunit (10), and an effective neuroprotectant for modulating NR2B receptor activity by changing the binding rate of the receptor (39). In the present study, ifenprodil showed specific antiepileptic effects by reducing PC neural excitability in patients with epilepsy and marginally increasing neural excitability in the patient without epilepsy. Furthermore, ifenprodil inhibited the neural network activity in brain tissue slices transformed from patients with MCD.

The limitations of the study are as follows: A small cohort of patients was used, which makes it challenging to avoid the bias brought by variables and in terms of the effect of ifenprodil, interneurons have not been involved in the current study. Therefore, further investigation for the expression, regulation, function of ifenprodil on interneurons is required.

In conclusion, although further studies are necessary to investigate whether ifenprodil exerts an effect on inhibitory interneurons and glial cells, the present findings suggest that ifenprodil may be utilized as a treatment method for epilepsy caused by MCD.

\section{Acknowledgements}

The present study was supported by the National Natural Science Foundation of China (grant no. 81571275).

\section{References}

1. Perucca E, French J and Bialer M: Development of new antiepileptic drugs: Challenges, incentives, and recent advances. Lancet Neurol 6: 793-804, 2007.

2. Aronica E, Becker AJ and Spreafico R: Malformations of cortical development. Brain Pathol 22: 380-401, 2012.

3. Vezzani A, Conti M, De Luigi A, Ravizza T, Moneta D, Marchesi F and De Simoni MG: Interleukin-1beta immunoreactivity and microglia are enhanced in the rat hippocampus by focal kainate application: Functional evidence for enhancement of electrographic seizures. J Neurosci 19: 5054-5065, 1999.

4. van Scheppingen J, Broekaart DW, Scholl T, Zuidberg MR, Anink JJ, Spliet WG, van Rijen PC, Czech T, Hainfellner JA, Feucht M, et al: Dysregulation of the (immuno)proteasome pathway in malformations of cortical development. J Neuroinflammation 13: 202, 2016.

5. Aronica E and Crino PB: Inflammation in epilepsy: Clinical observations. Epilepsia 3 (52 Suppl): S26-S32, 2011.

6. Zurolo E, Iyer A, Maroso M, Carbonell C, Anink JJ, Ravizza T, Fluiter K, Spliet WG, van Rijen PC, Vezzani A and Aronica E: Activation of Toll-like receptor, RAGE and HMGB1 signalling in malformations of cortical development. Brain 134: 1015-1032, 2011.

7. Boer K, Jansen F, Nellist M, Redeker S, van den Ouweland AM, Spliet WG, van Nieuwenhuizen O, Troost D, Crino PB and Aronica E: Inflammatory processes in cortical tubers and subependymal giant cell tumors of tuberous sclerosis complex. Epilepsy Res 78: 7-21, 2008.
8. Maroso M, Balosso S, Ravizza T, Liu J, Aronica E, Iyer AM, Rossetti C, Molteni M, Casalgrandi M, Manfredi AA, et al: Toll-like receptor 4 and high-mobility group box-1 are involved in ictogenesis and can be targeted to reduce seizures. Nat Med 16: 413-419, 2010.

9. Chiavegato A, Zurolo E, Losi G, Aronica E and Carmignoto G: The inflammatory molecules IL- $1 \beta$ and HMGB1 can rapidly enhance focal seizure generation in a brain slice model of temporal lobe epilepsy. Front Cell Neurosci 8: 155, 2014.

10. Chenard BL and Menniti FS: Antagonists selective for NMDA receptors containing the NR2B subunit. Curr Pharm Des 5: 381-404, 1999.

11. Fisher RS, Cross JH, D'Souza C, French JA, Haut SR, Higurashi N, Hirsch E, Jansen FE, Lagae L, Moshé SL, et al: Instruction manual for the ILAE 2017 operational classification of seizure types. Epilepsia 58: 531-542, 2017.

12. Hemb M, Velasco TR, Parnes MS, Wu JY, Lerner JT, Matsumoto JH, Yudovin S, Shields WD, Sankar R, Salamon N, et al: Improved outcomes in pediatric epilepsy surgery: The UCLA experience, 1986-2008. Neurology 74: 1768-1775, 2010.

13. Hoffman WH and Haberly LB: Bursting-induced epileptiform EPSPs in slices of piriform cortex are generated by deep cells. J Neurosci 11: 2021-2031, 1991.

14. Hoffman WH and Haberly LB: Bursting induces persistent all-or-none EPSPs by an NMDA-dependent process in piriform cortex. J Neurosci 9: 206-215, 1989.

15. Wang B, Yin L, Zou X, Ye M, Liu Y, He T, Deng S, Jiang Y, Zheng R, Wang Y, et al: A Subtype of inhibitory interneuron with intrinsic persistent activity in human and monkey neocortex. Cell Rep: Mar 3, 2015 (Epub ahead of print).

16. Viviani B, Bartesaghi S, Gardoni F, Vezzani A, Behrens MM, Bartfai T, Binaglia M, Corsini E, Di Luca M, Galli CL and Marinovich M: Interleukin-1beta enhances NMDA receptor-mediated intracellular calcium increase through activation of the Src family of kinases. J Neurosci 23: 8692-8700, 2003.

17. Geiger JR and Jonas P: Dynamic control of presynaptic $\mathrm{Ca}(2+)$ inflow by fast-inactivating $\mathrm{K}(+)$ channels in hippocampal mossy fiber boutons. Neuron 28: 927-939, 2000.

18. Connors BW, Gutnick MJ and Prince DA: Electrophysiological properties of neocortical neurons in vitro. J Neurophysiol 48: 1302-1320, 1982.

19. Lotze MT and Tracey KJ: High-mobility group box 1 protein (HMGB1): Nuclear weapon in the immune arsenal. Nat Rev Immunol 5: 331-342, 2005.

20. Wang L, Zhang X, Liu L, Cui L, Yang R, Li M and Du W: Tanshinone II A down-regulates HMGB1, RAGE, TLR4, NF-kappaB expression, ameliorates BBB permeability and endothelial cell function, and protects rat brains against focal ischemia. Brain Res 1321: 143-151, 2010.

21. Zhang R, Lao L, Ren K and Berman BM: Mechanisms of acupuncture-electroacupuncture on persistent pain. Anesthesiology 120: 482-503, 2014.

22. Bianchi ME and Manfredi AA: Immunology. Dangers in and out. Science 323: 1683-1684, 2009.

23. Bianchi ME: DAMPs, PAMPs and alarmins: All we need to know about danger. J Leukoc Biol 81: 1-5, 2007.

24. Catterall WA: Structure and regulation of voltage-gated $\mathrm{Ca} 2+$ channels. Annu Rev Cell Dev Biol 16: 521-555, 2000.

25. Huttunen HJ and Rauvala H: Amphoterin as an extracellular regulator of cell motility: From discovery to disease. J Intern Med 255: 351-366, 2004.

26. Pedrazzi M, Patrone M, Passalacqua M, Ranzato E, Colamassaro D, Sparatore B, Pontremoli S and Melloni E: Selective proinflammatory activation of astrocytes by high-mobility group box 1 protein signaling. J Immunol 179: 8525-8532, 2007.

27. Najm IM, Ying Z, Babb T, Mohamed A, Hadam J, LaPresto E, Wyllie E, Kotagal P, Bingaman W and Foldvary N: Epileptogenicity correlated with increased N-methyl-D-aspartate receptor subunit NR2A/B in human focal cortical dysplasia. Epilepsia 41: 971-976, 2000.

28. Thomas CG, Miller AJ and Westbrook GL: Synaptic and extrasynaptic NMDA receptor NR2 subunits in cultured hippocampal neurons. J Neurophysiol 95: 1727-1734, 2006.

29. Botta P, Demmou L, Kasugai Y, Markovic M, Xu C, Fadok JP, Lu T, Poe MM, Xu L, Cook JM, et al: Regulating anxiety with extrasynaptic inhibition. Nat Neurosci 18: 1493-1500, 2015. 
30. Waters $\mathrm{J}$ and Helmchen F: Background synaptic activity is sparse in neocortex. J Neurosci 26: 8267-8277, 2006.

31. Bean BP: The action potential in mammalian central neurons. Nat Rev Neurosci 8: 451-465, 2007.

32. Gardoni F, Boraso M, Zianni E, Corsini E, Galli CL, Cattabeni F, Marinovich M, Di Luca M and Viviani B: Distribution of interleukin-1 receptor complex at the synaptic membrane driven by interleukin-1 $\beta$ and NMDA stimulation. J Neuroinflammation 8 : 14, 2011.

33. García-Arnandis I, Guillén MI, Gomar F, Pelletier JP, Martel-Pelletier $\mathrm{J}$ and Alcaraz MJ: High mobility group box 1 potentiates the pro-inflammatory effects of interleukin- $1 \beta$ in osteoarthritic synoviocytes. Arthritis Res Ther 12: R165, 2010.

34. Howard P, Twycross R, Shuster J, Mihalyo M, Rémi J and Wilcock A: Anti-epileptic drugs. J Pain Symptom Manage 42: 788-804, 2011.
35. Neels HM, Sierens AC, Naelaerts K, Scharpé SL, Hatfield GM and Lambert WE: Therapeutic drug monitoring of old and newer anti-epileptic drugs. Clin Chem Lab Med 42: 1228-1255, 2004.

36. O'Neill LA and Bowie AG: The family of five: TIR-domain-containing adaptors in toll-like receptor signalling. Nat Rev Immunol 7: 353-364, 2007.

37. Wang CX and Shuaib A: NMDA/NR2B selective antagonists in the treatment of ischemic brain injury. Curr Drug Targets CNS Neurol Disord 4: 143-151, 2005.

38. Tu W, Xu X, Peng L, Zhong X, Zhang W, Soundarapandian MM, Balel C, Wang M, Jia N, Zhang W, et al: DAPK1 interaction with NMDA receptor NR2B subunits mediates brain damage in stroke. Cell 140: 222-234, 2010.

39. Perin-Dureau F, Rachline J, Neyton J and Paoletti P: Mapping the binding site of the neuroprotectant ifenprodil on NMDA receptors. J Neurosci 22: 5955-5965, 2002. 